01

\title{
Оценка статистически непредсказуемых изменений физических величин на больших интервалах наблюдения
}

\author{
() И.И. Горбань
}

Институт проблем математических машин и систем НАН Украины, 03187 Киев, Украина

e-mail: igor.gorban@yahoo.com

(Поступило в Редакцию 30 января 2018 г.)

Разработана новая методика оценки статистически непредсказуемых изменений физических величин на больших интервалах наблюдения. Методика основана на предположении, что имеют место медленные изменения измеряемой величины, среднее этих изменений статистически неустойчиво, а среднее статистически помехоустойчиво. Методика учитывает действие статистически неустойчивых, статистически устойчивых и детерминированных закономерностей. В тестовых примерах достигнуто повышение помехоустойчивости более чем на $20 \mathrm{~dB}$.

DOI: $10.21883 / J T F .2018 .12 .46777 .45-18$

\section{Введение}

Любая методика измерения (оценки) физической величины базируется на некоторых допущениях, служащих основой модели измерения.

Например, широко распространенная методика прямых измерений физических величин с многократными независимыми наблюдениями [1] основана на следующих предположениях:

1) за время проведения измерения $T$ измеряемая величина $s$ не изменяется;

2) после компенсации систематических погрешностей результаты $N$ однократных независимых наблюдений $x_{1}, x_{2}, \ldots, x_{N}$ в моменты $t_{1}, t_{2}, \ldots t_{N}$ времени $t$ описываются аддитивной моделью

$$
x_{n}=s_{n}+w_{n} \quad(n=\overline{1, N}),
$$

где $w_{n}-$ погрешность (помеха) случайного характера (характеризуемая некоторым определенным хотя, возможно, и неизвестным, законом распределения) с нулевым математическим ожиданием;

3) выборочное среднее $y=\frac{1}{N} \sum_{n=1}^{N} x_{n}$ результатов наблюдения обладает свойством сходимости к измеряемой величине $s$.

Согласно этой методике, за результат измерения принимают выборочное среднее $y_{N}$, а случайную погрешность оценивают средним квадратическим отклонением (СКО) результата измерения

$$
\sigma_{N}=\sqrt{\frac{1}{(N-1) N} \sum_{n=1}^{N}\left(x_{n}-y_{N}\right)^{2}} \cdot 1
$$

\footnotetext{
1 Формула (2) соответствует случаю, когда наблюдения $x_{1}, x_{2}, \ldots, x_{N}$ независимы и описываются одним и тем же законом распределения (помеха представляет собой белый шум, в общем случае не обязательно гауссовый).
}

Непрерывным аналогом описанной методики измерения является фильтрация наблюдаемого процесса

$$
x(t)=s+w(t)
$$

низкочастотным фильтром с верхней частотой пропускания $\Delta f=1 / T$ (рис. $1, a)$, где $w(t)-$ случайная помеха. В результате фильтрации отношение сигнал-помеха по мощности возрастает в $\rho=\int_{0}^{f_{e}} S_{w}(f) d f / \int_{0}^{\Delta f} S_{w}(f) d f$ раз, где $\left[0, f_{e}\right]$ - рабочая полоса, $S_{w}(f)$ - спектральная плотность мощности (СПМ) помехи. ${ }^{2}$

На малых интервалах измерения $T$ описанные модели измерения хорошо отражают реалии, однако оказываются не адекватными, когда время измерения велико и количество однократных наблюдений $N$ большое (исчисляется тысячами, десятками тысяч и более наблюдений).

На больших интервалах наблюдения реальные физические величины изменяется во времени. При этом задача оценки физической величины $s$ трансформируется в задачу оценки (фильтрации) физического процесса $s(t)$ (сигнала) по результатам наблюдения процесса при наличии помехи $w(t)$. В случае аддитивной помехи непрерывная модель измерения описывается выражением

$$
x(t)=s(t)+w(t),
$$

а дискретная модель - выражением

$$
x_{n}=s_{n}+w_{n} \quad(n=\overline{1, N}),
$$

где $s_{n}$ - значение оцениваемого процесса $s(t)$ в момент времени $t_{n}(n=\overline{1, N})$.

Известно много разных способов решения этой задачи. Все они используют те или иные отличия свойств сигнала от свойств помехи. Если отличий нет, задача оказывается не разрешимой.

\footnotetext{
2 В случае помехи в виде белого шума помехоустойчивость возрастает в $\rho=f_{e} / \Delta f$ раз.
} 
$a$
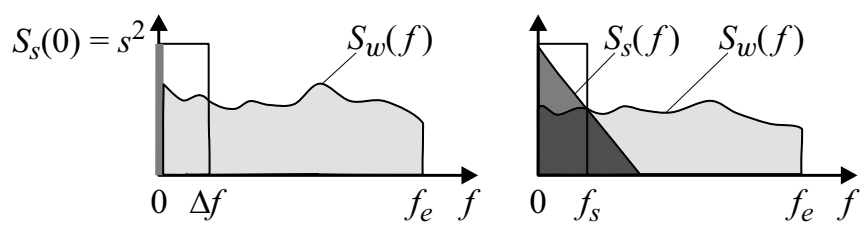

Рис. 1. СПМ измеряемой величины $S_{s}(f)$ и помехи $S_{w}(f)$ при отсутствии $(a)$ и наличии $(b)$ колебаний измеряемой величины.

Многие способы решения указанной задачи учитывают отличия статистических свойств сигнала и помехи и реализуются с помощью разнообразных вероятностных моделей.

До недавнего времени полагали, что реальные статистики (функции выборки) обладают свойством сходимости и потому на больших интервалах наблюдения применимы классические вероятностные модели, предполагающие сходимость статистик. Однако многочисленные экспериментальные исследования физического феномена статистической устойчивости показали, что возможности применения классических вероятностных моделей ограничены.

Выяснилось [2-7], что при небольшом объеме данных реальные статистики проявляют тенденцию к стабилизации, но при больших выборках тенденция стремления статистик к фиксированным величинам экспериментально не подтверждается. При увеличении объема выборки выборочная дисперсия, в частности, реального среднего $y_{N}$ результатов наблюдения $x_{1}, x_{2}, \ldots x_{N}$ вначале уменьшается. На этом этапе прослеживается тенденция стремления среднего $y_{N}$ к некоторой постоянной величине в соответствии с классическим законом больших чисел и наблюдается тенденция стремления выборочной функции распределения среднего $y_{N}$ к гауссовому распределению в соответствии с классической центральной предельной теоремой. Однако далее ситуация изменяется [2-5,7,8]: выборочная дисперсия среднего $y_{N}$ перестает уменьшаться, само среднее $y_{N}$ перестает проявлять тенденцию стремления к какой-либо постоянной величине, а его выборочная функция распределения перестает проявлять тенденцию стремления не то что к гауссовому, но вообще к какому-либо определенному закону распределения.

Теоретические и экспериментальные исследования показывают $[3,5,7,8]$, что при большом объеме данных действует обобщенный закон больших чисел, согласно которому среднее $y_{N}$ флуктуирует статистически непредсказуемо в определенном интервале, и справедлива обобщенная центральная теорема, согласно которой выборочная функция среднего флуктуирует в определенных границах, описываемых фрагментами гауссовых кривых.

Причин, вызывающих столь странное на первый взгляд поведение статистик, множество [2-5]. Установле- но $[2,3,7,9]$, что нарушение статистической устойчивости среднего связано с особенностями спектра рассматриваемого процесса. В частности, нарушение устойчивости имеет место, если в области низких частот СПМ процесса изменяется с частотой $f$ по закону $a / f^{\beta}$, где параметр формы спектра $\beta \geq 1$. Шумы, описываемые такой СПМ (фликкер-шумы) были обнаружены давно [10,11]. Они изучаются на протяжении уже почти ста лет [12,13]. Особый интерес к ним вызван тем, что подавить их путем усреднения результатов многократных наблюдений невозможно.

Результаты многочисленных экспериментальных исследований указывают на то [4,5], что реальный физический мир, по всей видимости, подчиняется трем типам закономерностей, а именно:

- детерминированным закономерностям, поддающимся описанию алгебраическими, дифференциальными и интегральными уравнениями;

- статистически прогнозируемым (статистически устойчивым) закономерностям, поддающимся описанию методами классической теории вероятностей и математической статистики, в основе которых лежит предположение о сходимости статистик;

- статистически непрогнозируемым (статистически неустойчивым) закономерностям, не поддающимся описанию классическими методами математической статистики.

На относительно небольших интервалах наблюдения обычно проявляются лишь первые два типа закономерностей, иногда по отдельности, иногда совместно. На больших и очень больших интервалах наблюдения существенную роль играют статистически непрогнозируемые закономерности, действующие в сочетании с детерминированными и статистически прогнозируемыми закономерностями.

Из сказанного следует, что при проведении измерений на больших интервалах наблюдения вместо моделей (1), (3) следует использовать модели (4), (5) и учитывать нарушения статистической устойчивости среднего множества наблюдений.

Во многих случаях нарушение статистической устойчивости среднего обусловлено исключительно медленными (т.е. низкочастотными) изменениями измеряемой физической величины. Иначе говоря, нарушение статистической устойчивости среднего $y(t)$ связано лишь сигналом $s(t)$; наличие же помехи не влияет на нарушение статистической устойчивости этого среднего.

Указанное отличие свойств сигнала и помехи можно использовать для подавления помехи и выделения сигнала на больших интервалах наблюдения. В работе [14] предложены общие принципы такого подхода и заложены основы новой методики измерения. Целью настоящей работы является уточнение и развитие этой методики, а также оценка эффективности ее применения на практике. 


\section{1. Идея методики измерения}

Новая методика оценки изменений физического процесса ориентирована, прежде всего, на совместный учет статистически устойчивых и статистически неустойчивых закономерностей. Эта методика базируется на следующих предположениях:

1) процесс $x(t)$, наблюдаемый на большом интервале $T$, слабо коррелирован и статистически неустойчив по отношению к среднему;

2) оцениваемый сигнал $s(t)$ представляет собой медленно протекающий статистически неустойчивый процесс длительностью $T$. В полосе сигнала $\left[0, f_{s}\right]$ его СПМ $S_{s}(f)=\frac{1}{T}|\dot{S}(f)|^{2}$ быстро спадает с повышением частоты (рис. $1, b)$, где $\dot{S}(f)$ - комплексный спектр сигнала;

$3)$ аддитивная помеха $w(t)$ представляет собой слабо коррелированный быстро флуктуирующий случайный процесс со статистически устойчивым средним и нулевым математическим ожиданием;

4) среднее СПМ помехи в полосе $\left[0, f_{s}\right]$ не превосходит средней СПМ $S_{w}(f)$ помехи в полосе $\left(f_{s}, f_{e}\right]$.

При этих достаточно общих предположениях можно оценить сигнал $s(t)$, не заботясь о том, какой конкретно вид он имеет и какой конкретно вид имеет закон распределения помехи $w(t)$.

Во всей рабочей полосе $\left[0, f_{e}\right]$ спектр $\dot{X}(f)$ наблюдаемого процесса $x(t)$ представляет собой сумму спектров сигнала $\dot{S}(f)$ и помехи $\dot{W}(f): \dot{X}(f)=\dot{S}(f)+\dot{W}(f)$. Однако при указанных предположениях определяющим в полосе $\left[0, f_{s}\right]$ является спектр сигнала $\dot{S}(f)$, а в полосе $\left[f_{s}, f_{e}\right]-$ спектр помехи $\dot{W}(f)$. Поэтому в качестве оценки сигнала можно использовать низкочастотную часть процесса $x(t)$.

Главная сложность практической реализации этой идеи состоит в корректном определении границы $f_{s}$, разделяющей спектр $\dot{X}(f)$ на низкочастотную $\dot{X}_{s}(f)$ и высокочастотную $\dot{X}_{w}(f)$ части.

Искомую границу $f_{s}$ грубо можно оценить на основе анализа амплитудного спектра $\dot{X}(f)$ (фиксации верхней границы интервала, на котором имеет место быстрое спадания спектра в области низких частот). Более точное значение этой границы можно найти из условия статистической устойчивости среднего $y_{w}(t)$ колебания $x_{w}(t)$, соответствующего высокочастотной части спектра $\dot{X}_{w}(f)$.

По низкочастотной части спектра $\dot{X}_{s}(f)$ можно рассчитать оценку полезного сигнала $s^{*}(t)$, а по высокочастотной части спектра $\dot{X}_{w}(f)-$ СКО погрешности измерения $\sigma^{*}$.

Прежде чем переходить к конкретному описанию методики, кратко остановимся на некоторых положениях теории гиперслучайных явлений ${ }^{3}[2-5,15]$, в рамках

\footnotetext{
3 Под гиперслучайным явлением подразумевается множество несвязанных между собой случайных явлений (событий, величин или функций), рассматриваемых как единое целое.
}

которой формализовано понятие статистической устойчивости и разработана методика фиксации нарушений статистической устойчивости и измерения интервалов статистической устойчивости различных статистик, в частности выборочного среднего.

\section{2. Статистическая устойчивость выборочного среднего на бесконечном интервале наблюдения}

Несмотря на широкое распространение понятия статистической устойчивости, как ни странно, до недавнего времени оно не было формализовано. Исследования показали, что статистическая устойчивость определяется особенностями процесса, его длительностью и типом статистики.

Нарушения статистической устойчивости можно зафиксировать с помощью параметров статистической неустойчивости. Формализация понятия статистической устойчивости базируется на этих параметрах.

При исследовании среднего случайной последовательности $X_{1}, X_{2}, \ldots, X_{N}$ часто используют параметр статистической неустойчивости $\gamma_{N}=\mathrm{E}\left[\bar{D}_{Y_{N}}\right] / \mathrm{E}\left[\bar{D}_{X_{N}}\right]$, где Е[.] - оператор математического ожидания, $\bar{D}_{Y_{N}}=$ $=\frac{1}{N-1} \sum_{n=1}^{N}\left(Y_{n}-\bar{m}_{Y_{N}}\right)^{2}-$ выборочная дисперсия флуктуации среднего $Y_{n}=\frac{1}{n} \sum_{i=1}^{n} X_{i}(n=\overline{2, N}), \bar{m}_{Y_{N}}=\frac{1}{N} \sum_{n=1}^{N} Y_{n}$ выборочное среднее флуктуации среднего, $\bar{D}_{X_{N}}=$ $=\frac{1}{N-1} \sum_{n=1}^{N}\left(X_{i}-Y_{N}\right)^{2}-$ выборочная дисперсия исходной последовательности.

Чем меньшие значения принимают параметр $\gamma_{N}$, тем более устойчивая последовательность по отношению к среднему. Случайные последовательности, у которых параметр $\gamma_{N}$ стремится к нулю при $N \rightarrow \infty$, считаются статистически устойчивыми по отношению к среднему. Последовательности же, которые таким свойством не обладают, считаются статистически неустойчивыми по отношению к среднему.

Для количественной оценки степени неустойчивости используют эталонную единицу измерения $\gamma_{0 N}$, представляющую собой параметр $\gamma_{N}$, рассчитанный для эталонной статистически устойчивой последовательности белого гауссового шума. Особенность этой единицы зависимость от объема выборки $N$. Аналитически она описывается выражением $[2,3] \gamma_{0 N}=\frac{N+1}{(N-1) N} C_{N}-\frac{2}{N-1}$, где $C_{N}=\sum_{n=1}^{N} 1 / n$.

Абсолютный уровень статистической неустойчивости по отношению к среднему в единицах измерения $\gamma_{0 N}$ характеризует параметр статистической неустойчивости $h_{N}=\gamma_{N} / \gamma_{0 N}$. 


\section{3. Статистическая устойчивость выборочного среднего на ограниченном интервале наблюдения}

При решении практических задач существенную роль играет не наличие или отсутствие сходимости среднего при бесконечно большом объеме выборки, а наличие или отсутствие тенденции сходимости среднего на рассматриваемом интервале наблюдения. При наличии такой тенденции процесс считается статистически устойчивым по отношению к среднему, в противном же случае статистически неустойчивым.

При формализации понятия „тенденция сходимости“ и при измерении интервала статистической устойчивости среднего $\tau$ используют границу статистической устойчивости среднего. Для параметра статистической неустойчивости $h_{N}$ эта граница определяется выражениями $h_{0 N}^{+}=\gamma_{0 N}^{+} / \gamma_{0 N}$, где $\gamma_{0 N}^{+}=\gamma_{0 N}+\varepsilon \sigma_{\gamma_{0 N}^{*}}, \varepsilon-$ параметр, характеризующий доверительный интервал, $\sigma_{\gamma_{0 N}^{*}}-$ СКО величины $\gamma_{0 N}^{*}=\bar{D}_{Y_{N}} / \mathrm{E}\left[\bar{D}_{X_{N}}\right]$, соответствующей эталонной статистически устойчивой последовательности. Аналитическая зависимость СКО $\sigma_{\gamma_{0 N}^{*}}$ от объема выборки $N$ описывается выражением [2,3]

$$
\begin{aligned}
& \sigma_{\gamma_{0 N}^{*}}=\frac{1}{N-1} \\
& \times \sqrt{\frac{2 C_{N}^{2}}{N^{2}}+\frac{(N+1) C_{N}}{N}+A_{N}\left(\frac{4}{N}-2\right)+\frac{8 B_{N}}{N}-12},
\end{aligned}
$$

где $A_{N}=\sum_{n=1}^{N} 1 / n^{2}, B_{N}=\sum_{n=1}^{N} C_{n-1} / n$.

Критерием нарушения статистической устойчивости по отношению к среднему, определяющим величину интервала статистической устойчивости $\tau$, служит выход параметра статистической неустойчивости $h_{N}$ за границу $h_{0 N}^{+}$статистической устойчивости.

При практических расчетах вместо параметра $h_{N}$ используют его оценку $h_{N}^{*}$, рассчитываемую для реализации $x_{1}, x_{2}, \ldots, x_{N}$ по формуле: $h_{N}^{*}=\gamma_{N}^{*} / \gamma_{0 N}$, где $\gamma_{N}^{*}=\bar{D}_{y_{N}} / \bar{D}_{x_{N}}$.

\section{4. Методика измерения}

Описываемая ниже методика предназначена для измерения (оценивания) физического процесса, наблюдаемого на интервале длительностью $T$, при условии выполнения предположений, изложенных в разд. 1. Эта методика включает следующие этапы.

1) Проводят дискретизацию наблюдаемого процесса $x(t)$ с равномерным шагом дискретизации $\Delta t$ (формируют выборку $(T=N \Delta t))$.

2) Оценивают величину $\tau^{*}$ интервала статистической устойчивости процесса $x(t)$ (определяют величину интервала наблюдения, при котором оценка параметра статистической неустойчивости $h_{N}^{*}$ достигает границы $h_{0 N}^{*}$ ).
3) Если оценка $\tau^{*} \ll T$, переходят к п. 4. В противном случае применяют стандартную методику измерения с использованием формул (1)-(2).

4) Вычисляют физический дискретный спектр $\dot{X}_{k}$ процесса $x_{1}, x_{2}, \ldots, x_{N}$, где $k$ - номер спектрального отсчета $(k=\overline{1, N / 2})$.

5) В области низких частот спектра $\left|\dot{X}_{k}\right|$ находят максимальный спектральный отсчет $k=k_{s}$, удовлетворяющий следующему условию: в полосе $\left[1, k_{s}\right]$ с увеличение номера спектрального отсчета $k$ амплитудный спектр $\left|\dot{X}_{k}\right|$ уменьшается в среднем быстрее, чем $1 / \sqrt{k}$.

6) Из физического спектра $\dot{X}_{k}$ вырезают высокочастотную часть $\dot{X}_{k w}$ (соответствующую полосе $\left.\left(k_{s}, N / 2\right]\right)$, проводят ее гетеродинирование в область нулевых частот и по полученному в результате гетеродинирования спектру $\dot{X}_{k w}^{\prime}$ рассчитывают оценку помехи $w_{1}^{*}, w_{2}^{*}, \ldots, w_{N}^{*}$.

7) Оценивают величину $\tau^{*}$ интервала статистической устойчивости оценки помехи $w_{1}^{*}, w_{2}^{*}, \ldots, w_{N}^{*}$ согласно п. 2. Если полученная оценка близка к величине интервала наблюдения $T$, то переходят к п. 8. В противном случае расширяют полосу $\left[1, k_{s}\right]$ и повторяют п. 6,7 .

8) По низкочастотной части $\dot{X}_{k s}$ спектра $\dot{X}_{k}$ процесса $x_{1}, x_{2}, \ldots, x_{N}$, соответствующей полосе $\left[1, k_{s}\right]$, рассчитывают оценку сигнала $s_{1}^{*}, s_{2}^{*}, \ldots, s_{N}^{*}$.

9) Используя оценку помехи $w_{1}^{*}, w_{2}^{*}, \ldots, w_{N}^{*}$ или спектр $\dot{X}_{k w}$, рассчитывают СКО погрешности измерения

$$
\sigma^{*}=\sqrt{\frac{2 k_{s}}{(N-1) N} \sum_{n=1}^{N} w_{n}^{* 2}}=\sqrt{\frac{4 k_{s}}{N^{3}} \sum_{k=k_{s}+1}^{N / 2}\left|\dot{X}_{k}\right|^{* 2}} .
$$

10) Задавшись параметром $\varepsilon$, определяющим величину доверительного интервала, находят результат измерения в виде интервальной величины $\tilde{\varepsilon}_{n}=\left[\varepsilon_{n}^{*}-\varepsilon \sigma_{n}, s_{n}^{*}+\varepsilon \sigma^{*}\right], n=\overline{1, N}$.

Используя оценку помехи $w_{1}^{*}, w_{2}^{*}, \ldots, w_{N}^{*}$ и СКО $\sigma^{*}$, можно оценить средний выигрыш в помехоустойчивости:

$$
\rho=\sum_{n=1}^{N} w_{n}^{* 2} /\left(N \sigma^{* 2}\right)=(N-1) /\left(2 k_{s}\right) \approx N /\left(2 k_{s}\right) .
$$

\section{5. Пример применения методики для измерения колебаний магнитного поля Земли}

Для выяснения эффективности применения предложенного подхода проведен ряд измерений различных физических процессов. В частности, были оценены статистически непредсказуемые изменения магнитного поля Земли. Исходные данные взяты с сайта [16], описывающие с дискретность 5 ч изменение магнитного поля в районе Москвы с 1997 по 2009 г. (рис. 2,a).

Соответствие исходных данных основным требованиям по применению методики иллюстрируют рис. 3 и 4. В частности, факт слабой корреляции элементов выборки демонстрирует рис. $3, a$, быстрое спадание спектра с 

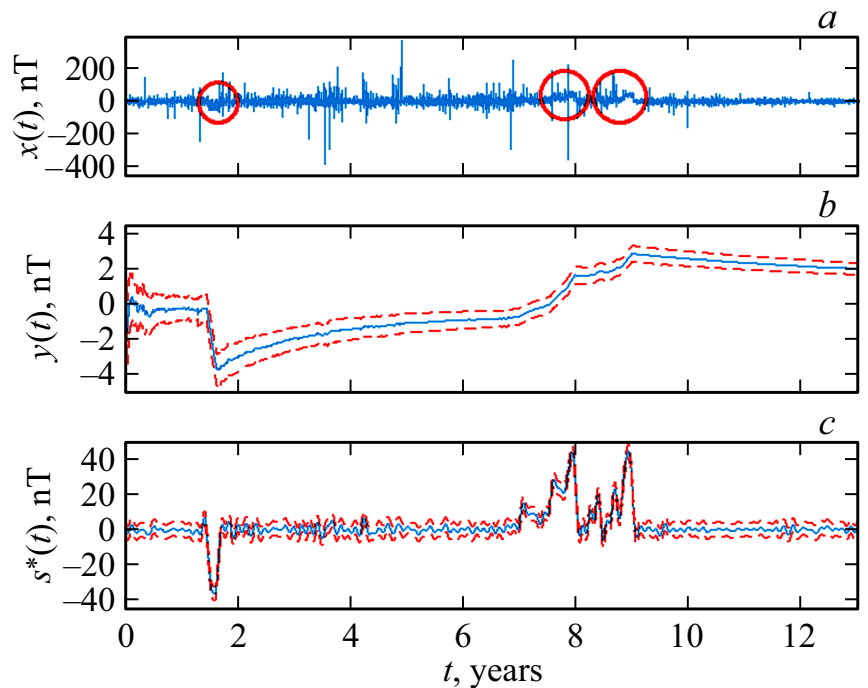

Рис. 2. Наблюдаемое колебание магнитного поля $(a)$, среднее этого колебания $(b)$ и оценка сигнала $(c)$. Штриховыми линиями изображены границы доверительного интервала, соответствующие $=3$.
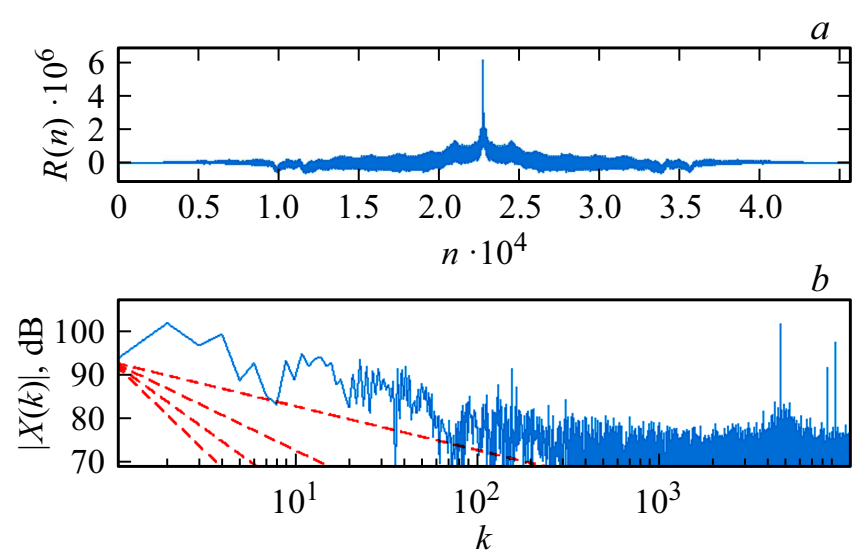

Рис. 3. Автокорреляционная функция наблюдаемого колебания $(a)$ и его амплитудный спектр $(b)$. Для сравнения на рис. $3, b$ штриховыми прямыми линиями изображены сдвинутые вдоль оси ординат до уровня первого отсчета спектра наблюдаемого колебания графики степенных функций $1 / k^{\beta / 2}$, $\beta=\overline{1,4}$.

повышение частоты в области низких частот (примерно до сотого спектрального отсчета) - рис. $3, b$, а нарушение статистической устойчивости среднего (фиксируемые в районе двух лет наблюдения) - рис. 4, $a$.

Наблюдаемое колебание явно не стационарно (рис. 5,a). Из-за нарушения статистической устойчивости среднего его выборочное среднее не проявляет стремления к фиксированной величине (рис. 5, $b, 6, a$ ), a его выборочная дисперсия среднего не проявляет тенденции монотонного уменьшения (рис. $5, b, 6, b$ ), что свидетельствует о наличии закономерностей, не описываемых классическим законом больших чисел и классической центральной теоремой.
Свойства же оценок помехи, рассчитанных на основе спектральных составляющих, превышающих сотый спектральный отсчет исследуемого процесса, иные. Выборочное среднее среднего оценки помехи проявляет стремление к стабилизации в соответствии с классическим законом больших чисел (рис. $5, c, 6, c$ ), а выборочная дисперсия среднего оценки помехи - тенденцию уменьшения в соответствии с классической центральной теоремой (рис. 5, $c, 6, d$ ).

Результат измерения магнитного поля по предлагаемой методике представлен на рис. 2,c. Для оценки

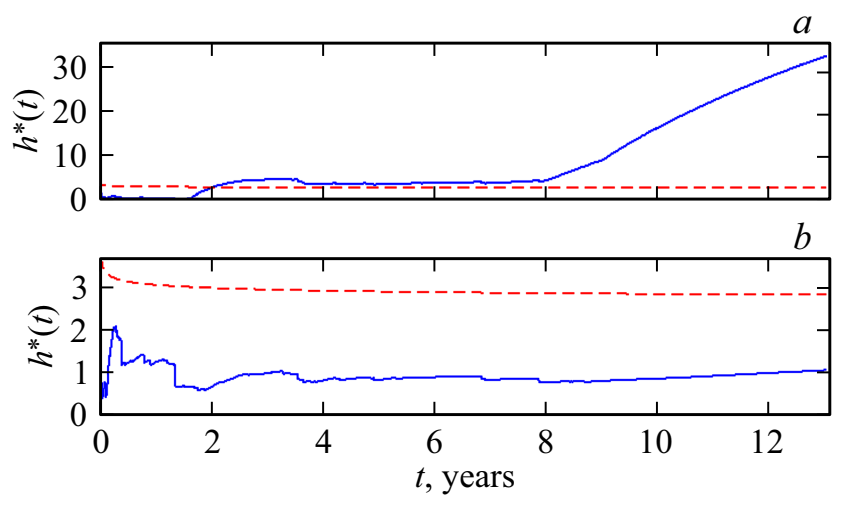

Рис. 4. Зависимости оценок параметра статистической неустойчивости $h^{*}(t)$ от времени наблюдения (непрерывные линии) для наблюдаемого колебания $(a)$ и оценки помехи $(b)$. Штриховыми линиями изображены верхние границы устойчивости.
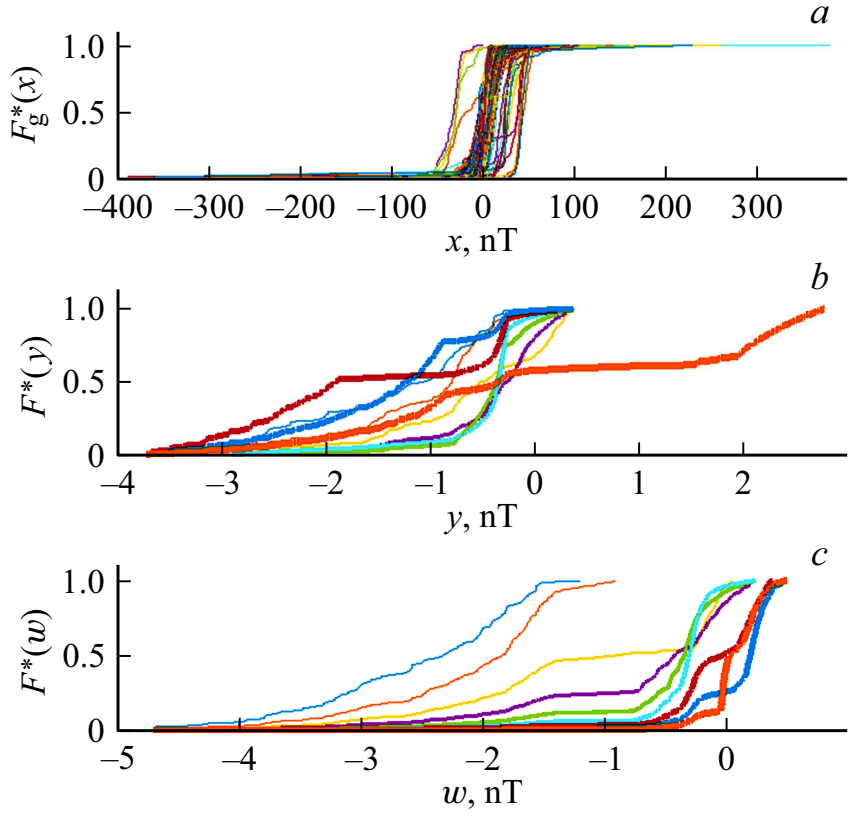

Рис. 5. Оценки функции распределения для 256 прилегающих друг к другу фрагментов наблюдаемого колебания $(a)$, оценки функции распределения для выборочного среднего длиной $256 \cdot 2^{(r-1)}(r=\overline{1,9})$ отсчетов наблюдаемого колебания $(b)$ и помехи $(c)$ (на двух последних рисунках толщина кривых возрастает с увеличением параметра $r$ ). 


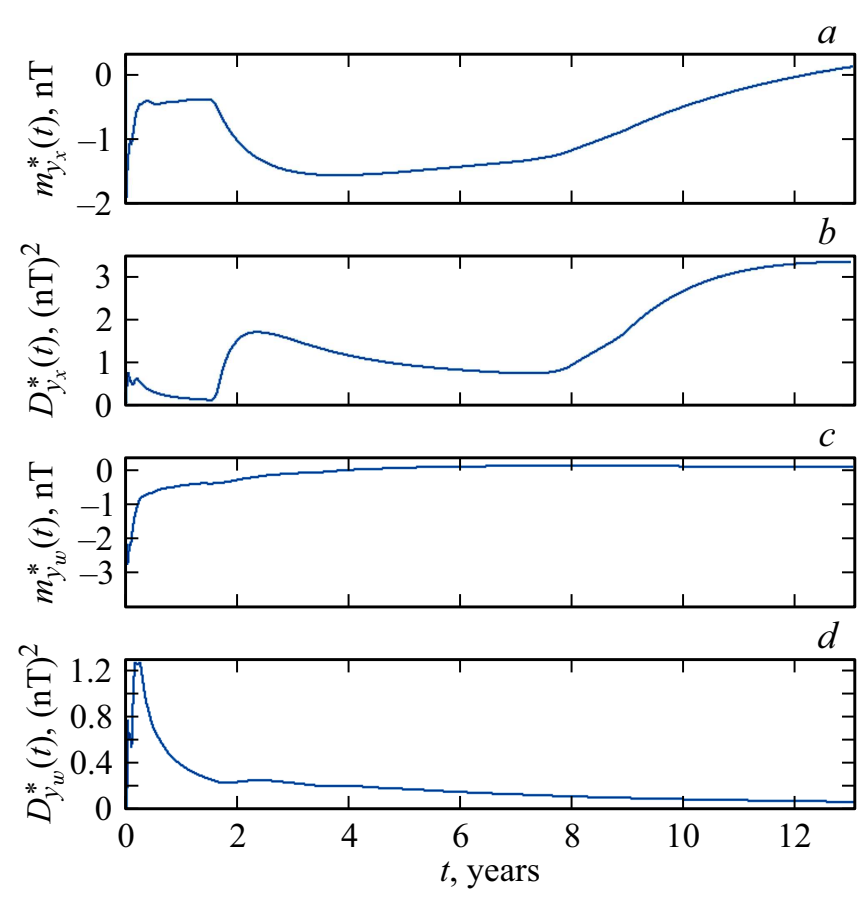

Рис. 6. Выборочное среднее среднего $(a, c)$ и выборочная дисперсия среднего $(b, d)$ наблюдаемого колебания $(a, b)$ и оценки помехи $(c, d)$.

сигнала использованы сто низкочастотных спектральных отсчетов наблюдаемого колебания.

Для сравнения на рис. $2, b$ приведены результаты измерения магнитного поля по стандартной методике, описываемой моделью (3), игнорирующей нарушение статистической устойчивости.

Анализируя колебание, изображенное на рис. 2, $a$, можно обнаружить в районе 1.6 года, 7.9 лет и 9 лет от начала наблюдения отклонения магнитной индукции на десятки единиц, сохраняющиеся на протяжении нескольких месяцев (участки колебания, выделенные на рисунке окружностями). Эти отклонения, с трудом обнаруживаемые на рис. 2, $a$, хорошо видны как на рис. $2, b$, так и на рис. $2, c$.

Однако сравнение результата измерения по стандартной методике (см. рис. $2, b)$ с результатом по новой методике (см. рис. 2,c) демонстрирует явное преимущество последней. Стандартная методика дает неприемлемо искаженный результат измерения. Фиксируемые на рис. $2, a$ отклонения индукции на уровне десятков единиц искаженно представлены на рис. $2, b$ отклонениями в несколько единиц. При этом эти отклонения сопровождаются длительными переходными процессами, отсутствующими на рис. 2, $a$.

Новая же методика отслеживает динамику изменения среднего уровня колебания, фиксируя при этом в районе 1.6 года, 7.9 лет и 9 лет отклонения индукции до $\pm 40 \mathrm{nT}$.

Расчеты показывают, что в данном случае в результате применения методики отношение сигнал-помеха возросло на $20.5 \mathrm{~dB}$.

\section{6. Обобщение методики}

Описанная методика измерения статистически непредсказуемых изменений физической величины на больших интервалах наблюдения учитывает действие статистически устойчивых и статистически неустойчивых закономерностей при слабой корреляции отсчетов выборки.

При сильной корреляции процесс необходимо декоррелировать. Часто эту процедуру можно свести к прореживанию отсчетов, однако такой прием не всегда эффективен. Если наблюдаемый процесс $x(t)$ содержит детерминированную составляющую циклического характера (например, связан с сезонными колебаниями), прореживание не приносит желаемого результата.

Систематическую погрешность, вызываемую циклическими колебаниями, можно подавить, вычисляя оценки математического ожидания $\hat{m}_{x}(t)$ и СКО $\hat{\sigma}_{x}(t)$ путем усреднения данных по ансамблю циклов (в некоторых случаях и путем дополнительного сглаживания по времени), а затем формируя центрированный и нормированный процесс

$$
\hat{x}(t)=\left(x(t)-\hat{m}_{x}(t)\right) / \hat{\sigma}_{x}(t) .
$$

Искомые оценки сигнала и погрешности могут быть рассчитаны на основе результатов оценивания этого процесса $\hat{x}(t)$.

Измерение при этом сводится к следующему:

1) наблюдаемый процесс представляют множеством отсчетов $x_{i j}, i-$ номер отсчета в цикле $(i=\overline{1, I}), j-$ номер цикла $(j=\overline{1, J})$;

2) рассчитывают оценку математического ожидания $m_{i}^{*}=\sum_{j=1}^{J} x_{i j} / J \quad$ и $\quad$ оценку дисперсии $D_{i}^{*}=\sum_{j=1}^{J}\left(x_{i j}-m_{i}^{*}\right)^{2} /(J-1) \quad$ путем $\quad$ усреднения $\quad$ по ансамблю;

3) формируют периодическую оценку математического ожидания $m_{i j}^{*}=m_{i}^{*}$ и оценку дисперсии $D_{i j}^{*}=D_{i}^{*}$ $(i=\overline{1, I}, j=\overline{1, J})$;

4) формируют сглаженные оценки математического ожидания $\hat{m}_{i j}=A_{i} * m_{i}^{*}$, дисперсии $\hat{D}_{i j}=A_{i} * D_{i}^{*}$ и СКО $\hat{\sigma}_{i j}=\sqrt{\hat{D}_{i j}}(i=\overline{1, I}, j=\overline{1, J})$, где $A_{i}-$ окно сглаживания, $*$ - оператор круговой свертки;

5) формируют центрированную и нормированную выборку $\hat{x}_{i j}=\left(x_{i j}-\hat{m}_{i j}\right) / i j$;

6) для центрированной и нормированной выборки проводят расчет согласно методике, описанной в разд. 4. В результате получают оценку для центрированного и нормированного сигнала в виде интервальной величины $\hat{\tilde{s}}_{i j}=\left[\hat{s}_{i j}-\varepsilon \hat{\sigma}, \hat{s}_{i j}+\varepsilon \hat{\sigma}\right](i=\overline{1, I}, j=\overline{1, J})$;

7) находят результат измерения в виде интервальной величины $\tilde{s}_{i j}=\left[s_{i j}^{*}-\varepsilon \sigma_{i j}^{*}, s_{i j}^{*}+\varepsilon \sigma_{i j}^{*}\right](i=\overline{1, I}, j=\overline{1, J})$, где $s_{i j}^{*}=\hat{s}_{i j} \hat{\sigma}_{i j}+\hat{m}_{i j}, \sigma_{i j}^{*}=\hat{\sigma} \hat{\sigma}_{i j}$. 

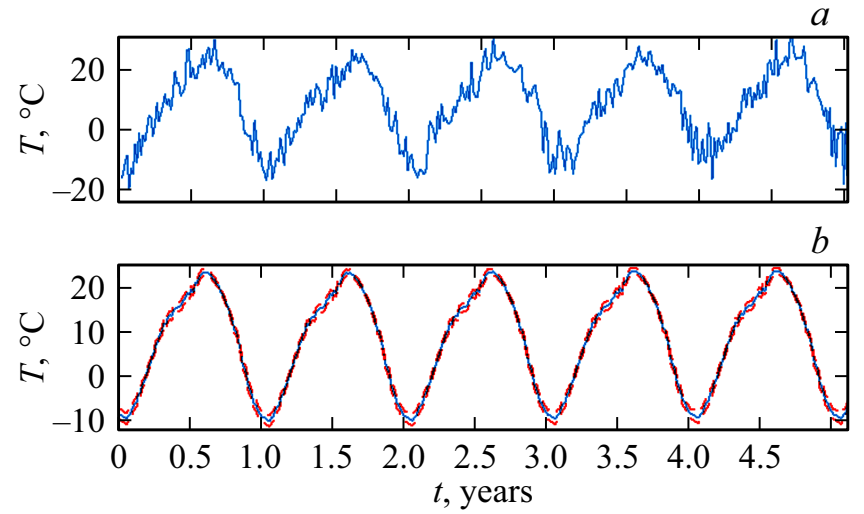

Рис. 7. Прореженное колебание максимальной суточной температуры воздуха за 1917-1921 г. во Владивостоке $(a)$ и оценка тренда (сигнала) $(b)$. Штриховыми линиями изображены границы доверительного интервала, соответствующие $\varepsilon=3$.

\section{7. Пример применения методики для оценки тренда изменения температуры воздуха}

Для выяснения эффективности применения обобщенной методики проведен ряд измерений различных физических процессов циклического типа. В частности, был оценен тренд колебания максимальной суточной температуры воздуха во Владивостоке за 92 года наблюдения с 1917 по 2008 г. Исходные данные взяты с сайта [17].

Выборка оказалась сильно коррелированной. С целью декорреляции для расчетов использован каждый пятый отсчет. Фрагмент прореженной выборки за первые 5 лет наблюдения представлен на рис. 7, $a$. На рис. 7, $b$ приведена оценка тренда, полученная с помощью описанной в п. 6 модификации методики.

Оценка тренда формировалась путем центрирования и нормирования прореженной выборки, а затем фильтрации сигнала с использованием первых двадцати спектральных отсчетов. Расчет оценок математического ожидания и дисперсии для центрирования и нормирования данных и расчет оценки погрешности измерения осуществлялись путем усреднения данных по 92 годам наблюдения. Применение описанной методики привело к повышению отношения сигнал-помеха на $22 \mathrm{~dB}$.

\section{Заключение}

На больших интервалах наблюдения реальные физические величины изменяются во времени, причем, как правило, статистически непредсказуемым образом. Эти изменения проявляются в нарушении статистической устойчивости статистик, в частности, выборочных средних наблюдаемых колебаний.

Статистическая устойчивость статистик зависит от особенностей спектра процесса. Нарушения статистической устойчивости среднего имеют место, если в низкочастотной области спектра процесса спектральная плотность мощности быстро спадает с увеличением частоты.

Стандартные методы измерения с многократными наблюдениями не учитывают факт изменения физической величины за время измерения и происходящие при этом нарушения статистической устойчивости среднего. Поэтому область применения стандартных методов измерения ограничена относительно небольшими интервалами наблюдения, на которых измеряемая величина остается практически постоянной, а нарушения статистической устойчивости среднего пренебрежимо малы. На практике это имеет место, когда объемы выборки невелики (не превосходят нескольких сотен или тысяч наблюдений).

При больших интервалах наблюдения и больших объемах выборки, исчисляемых тысячами, десятками тысяч и более отсчетов, задача оценки физической величины трансформируется в задачу оценки физического процесса (сигнала) по результатам регистрации наблюдаемого процесса.

Разработанная новая методика оценки на больших интервалах наблюдения статистически непредсказуемых изменений физических величин основана на предположении, что энергия оцениваемого сигнала сосредоточена в области низких частот, энергия аддитивной помехи в высокочастотной области, среднее сигнала статистически неустойчиво, а среднее помехи статистически устойчиво. Методика учитывает действие статистически неустойчивых, статистически устойчивых и детерминированных закономерностей.

Ключевой вопрос в этой методике - нахождение границы, разделяющей спектр наблюдаемого процесса на низкочастотную и высокочастотную части. Эта задача решается методами физико-математической теории гиперслучайных явлений.

На тестовых примерах продемонстрирована высокая эффективность применения новой методики (достигнуто повышение отношения сигнал-помеха более чем на $20 \mathrm{~dB})$.

\section{Список литературы}

[1] ГОСТ 8.207-76. Прямые измерения с многократными наблюдениями. М.: ИПК Издательство стандартов, 2001. $8 \mathrm{c}$.

[2] Горбань И.И. Феномен статистической устойчивости. К: Наукова думка, 2014. 444 c.

[3] Gorban I.I. The statistical stability phenomenon. Springer, 2017. $362 \mathrm{p}$.

[4] Горбань И.И. Случайность и гиперслучайность. К.: Наукова думка, 2016. 288 с.

[5] Gorban I.I. Ramdomness and hyper-randomness. Springer, 2018. $256 \mathrm{p}$.

[6] Еськов В.В., Гавриленко Т.В., Еськов В.М. и др. // ЖТФ. 2017. Т. 87. Вып. 11. С. 1609-1614.

[7] Горбань И.И. // ЖТФ. 2014. Т. 59. Вып. 3. С. 22-30.

[8] Горбань И.И. // Системные исследования и информационные технологии. 2015. № 4. С. 207-210. 
[9] Горбань И.И. // Известия вузов. Радиоэлектроника. 2012. T. 55. № 3. C. 3-18.

[10] Johnson J.B. // Phys. Rev. 1925. Vol. 26.

[11] Schottky W. // Phys. Rev. 1926. Vol. 28.

[12] Коган Ш.М. // УФН. 1985. Т. 145. № 2. С. 285-325.

[13] Жигальский Г.П. // УФН. 2003. Т. 173. № 5. С. 465-490.

[14] Горбань И.И. // Математические машины и системы. 2017. № 4. С. 104-111.

[15] Горбань И.И. // Проблемы управления и информатики. 2017. № 4. С. 19-35.

[16] Данные о вариации индукции магнитного поля в районе Москвы. Электронный ресурс. Режим доступа: http://forecast.izmiran.rssi.ru/bankr.htm

[17] Данные о погоде во Владивостоке. Электронный ресурс. Режим доступа:

$\mathrm{http} / / /$ thermo.karelia.ru/weather/w_history.php 